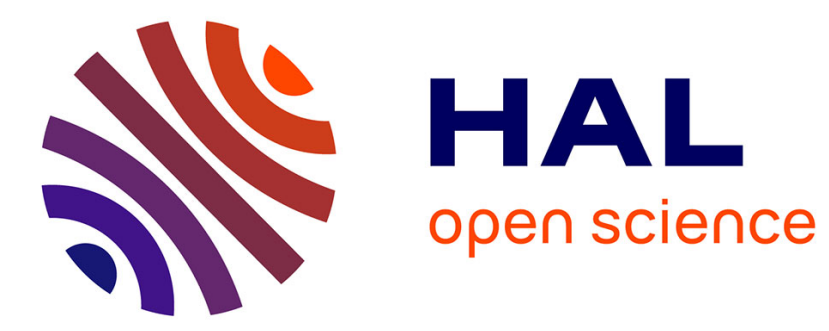

\title{
A geometric interpretation of coherent structures in Navier-Stokes flows
}

\author{
Ian Roulstone, Bertrand Banos, J. Gibbon, Vladimir Roubtsov
}

\section{To cite this version:}

Ian Roulstone, Bertrand Banos, J. Gibbon, Vladimir Roubtsov. A geometric interpretation of coherent structures in Navier-Stokes flows. Proceedings of Royal Society A Mathematical, physical and engineering sciences, 2009, 465 (2107), pp.2015 - 2021. 10.1098/rspa.2008.0483 . hal-03054038

\section{HAL Id: hal-03054038 \\ https://univ-angers.hal.science/hal-03054038}

Submitted on 11 Dec 2020

HAL is a multi-disciplinary open access archive for the deposit and dissemination of scientific research documents, whether they are published or not. The documents may come from teaching and research institutions in France or abroad, or from public or private research centers.
L'archive ouverte pluridisciplinaire $\mathbf{H A L}$, est destinée au dépôt et à la diffusion de documents scientifiques de niveau recherche, publiés ou non, émanant des établissements d'enseignement et de recherche français ou étrangers, des laboratoires publics ou privés. 


\title{
PROCEEDINGS
}

\section{A geometric interpretation of coherent structures in Navier-Stokes flows}

\author{
By I. Roulstone ${ }^{1, *}$, B. Banos ${ }^{2}$, J. D. Gibbon ${ }^{3}$ and V. N. Roubtsov ${ }^{4,5}$ \\ ${ }^{1}$ Department of Mathematics, University of Surrey, Guildford GU2 7XH, UK \\ ${ }^{2}$ Lycée Chaptal de Saint Brieuc, 6 allée Chaptal, 22 000 Saint-Brieuc, France \\ ${ }^{3}$ Department of Mathematics, Imperial College London, London SW7 2AZ, UK \\ ${ }^{4}$ Départment de Mathématiques, Université d'Angers, 2 Boulevard Lavoisier, \\ 49045 Angers, France \\ ${ }^{5}$ Theory Division, ITEP, 25, Bolshaja Tcheremushkinskaya, Moscow 117259, Russia
}

The pressure in the incompressible three-dimensional Navier-Stokes and Euler equations is governed by Poisson's equation: this equation is studied using the geometry of threeforms in six dimensions. By studying the linear algebra of the vector space of three-forms $\Lambda^{3} W^{*}$ where $W$ is a six-dimensional real vector space, we relate the characterization of non-degenerate elements of $\Lambda^{3} W^{*}$ to the sign of the Laplacian of the pressure -and hence to the balance between the vorticity and the rate of strain. When the Laplacian of the pressure, $\Delta p$, satisfies $\Delta p>0$, the three-form associated with Poisson's equation is the real part of a decomposable complex form and an almost-complex structure can be identified. When $\Delta p<0$, a real decomposable structure is identified. These results are discussed in the context of coherent structures in turbulence.

\section{Keywords: Navier-Stokes equations; three-forms; turbulence;} almost-complex structures

\section{Equations for an incompressible fluid}

It is rare in fluid dynamics for highly technical abstract geometrical criteria to have a direct correspondence with experimental observations. In a seminal paper, Douady et al. (1991; henceforth DCB) devised an experimental method based on the cavitation in a liquid seeded with bubbles to observe regions of low pressure corresponding to regions of highly concentrated vorticity. For the incompressible Navier-Stokes or Euler equations (where viscosity $\nu=0$ )

$$
\boldsymbol{u}_{t}+\boldsymbol{u} \cdot \nabla \boldsymbol{u}=\nu \Delta \boldsymbol{u}-\nabla p
$$

the incompressibility constraint $\operatorname{div} \boldsymbol{u}=0$ enforces the relation

$$
\Delta p=-u_{i, j} u_{j, i}=\frac{1}{2} \zeta^{2}-\operatorname{Tr} S^{2}
$$

where $S$ is the rate of strain matrix; $\boldsymbol{\zeta}=\nabla \times \boldsymbol{u}$ is the vorticity; $u_{i, j}=\partial u_{i} / \partial x_{j}$; and the summation convention is used. DCB established an analogy with electrostatics; namely that ' $p$ corresponds to the potential resulting from

* Author for correspondence (i.roulstone@surrey.ac.uk). 
negative and positive charges distributed in proportion to the square of the vorticity and the energy dissipation, respectively'.

Detailed computations of the three-dimensional incompressible Navier-Stokes equations illustrate the phenomenon exploited experimentally by DCB that vorticity has a tendency to accumulate on 'thin sets' whose morphology is characterized by quasi-one-dimensional tubes or filaments and quasi-twodimensional sheets. This description is in itself approximate as these thin structures undergo dramatic morphological changes in time and space. The topology is highly complicated; sheets tend to roll up into tube-like structures, while tubes tangle and knot akin to spaghetti boiling in a pan. The first vortex tubes $^{1}$ visualized in black and white were seen by Siggia (1981), and in colour by Kerr (1985). Vortex tubes usually have a short lifetime, vanishing at one place and reforming at another and correspond to regions of low pressure. Vorticity has a tendency to concentrate on these thin sets and it is within these where vorticity dominates strain, whereas the opposite is true outside of them. Thus, $\Delta p>0$ within, whereas outside $\Delta p<0$. Some theoretical reasons why these thin sets exist has recently been discussed by Gibbon (2008b).

In this paper, it is shown how a change in sign of the Laplacian may be interpreted in terms of the geometry of three-forms on a six-dimensional real vector space; equation (1.2), in which time is a parameter, is the basis of our geometric arguments and the conclusions reached are valid for both the Navier-Stokes and Euler equations. It is, of course, to be expected that any geometric structure should be independent of viscosity. From now on when we refer to the Navier-Stokes equations it should be implicitly understood that the Euler equations are also included.

The work of Roubtsov \& Roulstone $(1997,2001)$ has shown how quaternionic and hyper-Kähler structures emerge in models of nearly geostrophic flows in atmosphere and ocean dynamics. These results were based on earlier work by McIntyre and Roulstone, and were reviewed by them in McIntyre \& Roulstone (2002). It has also been shown that the three-dimensional Euler equations has a quaternionic structure in the dependent variables (Gibbon 2002) and that this idea can be used to discuss the evolution of orthonormal frames on particle trajectories: see Gibbon et al. (2006), Gibbon (2007, 2008a) and Gibbon \& Holm (2007). The use of different sets of dependent and independent variables in geophysical models of cyclones and fronts has facilitated some remarkable simplifications of otherwise hopelessly difficult nonlinear problems: see Hoskins \& Bretherton (1972). Roulstone \& Sewell (1997) and McIntyre \& Roulstone (2002), describe how contact and Kähler geometries provide a framework for understanding the basis of the various coordinate transformations that have proven so useful in this context. This present work has evolved from that of Roubtsov \& Roulstone (2001) using the results of Banos (2002). ${ }^{2}$ We note that the MongeAmpère structures developed by Lychagin et al. (1993), Banos (2002) and Kushner et al. (2007) are not present when (1.2) is studied in the context of the

\footnotetext{
${ }^{1}$ The visualizations by Vincent \& Meneguzzi (1994) were mainly of vortex sheets.

${ }^{2}$ Our initial results arose from our interest in Kähler geometry and Monge-Ampère equations in two independent variables (Roulstone et al. in press). It is well known that (1.2) gives rise to a Monge-Ampère equation in two-dimensional incompressible flow (Larchevêque 1990, 1993), which is elliptic when $\Delta p>0$. It is straightforward to show, using the formalism of Lychagin et al. (1993), that an almost-complex structure can be associated with this elliptic equation.
} 
incompressible Navier-Stokes equations in three dimensions. However, the representation of (1.2) in terms of three-forms on the six-dimensional phase space of the fluid (i.e. $T^{*} \mathbb{R}^{3}$, with local coordinates $\left(x_{i}, u_{i}\right)$ ) can be studied using the results of Hitchin (2000) upon which Banos' paper is based.

Banos (2002, definition 4.1) has shown how the generalized complex geometry of Hitchin and its real counterpart can be associated with elliptic and hyperbolic Monge-Ampère structures in three dimensions. In this study of turbulent flows in three dimensions, we show how regions in which $\Delta p>0$ (referred to as 'elliptic' by Weiss (1991) in the context of two-dimensional flows) are characterized by almostcomplex structures in the sense of Hitchin (2000) and Banos (2002). In a similar fashion, regions in which $\Delta p<0$ (referred to as 'hyperbolic' by Weiss 1991) can be characterized by real structures.

Fluid dynamicists may find the description of exterior calculus given in $\S 5$ of McIntyre \& Roulstone (2002) and the text by Kushner et al. (2007) useful in explaining the geometry of (1.2) in terms of (2.3). The text by McDuff \& Salamon (1998) is an excellent introduction to differential geometry on complex manifolds. Our notation follows the conventions set out in these references and in Hitchin (2000).

\section{The geometry of three-forms and Navier-Stokes flows in three dimensions}

One interesting feature of Navier-Stokes flows is the fact that the thin sets discussed in $\$ 1$ corresponding to quasi-one- and -two-dimensional topologies are also the manifestation of exact solutions of the Navier-Stokes equations. Known as Burgers' vortices (Burgers 1948), these correspond to either straight tubes or flat sheets depending on whether stretching is chosen in one or two directions (see Moffatt et al. 1994; Gibbon et al. 1999). These exact solutions are highly idealized, whereas computations and experiments show that the reality is closer to a tangle of spaghetti. Nevertheless, given the ubiquity of such flows, it is reasonable to think of them as an attracting class of low-dimensional solutions. It could be speculated that the intense bending, tangling and stretching they undergo may simply mean that these solutions may move onto complex manifolds of a higher dimension (cf. Roulstone et al. in press). One of the simplest of these is the CalabiYau manifold. These are smooth complex manifolds with a Ricci-flat Kähler metric and a holomorphically trivial canonical bundle.

The formulation of the geometry required can be found in Hitchin (2000) and Banos (2002), and references therein; note that Banos (2002) §3.2 casts Hitchin's results in the context of a symplectic vector space, which is what we work with here. Let $W$ denote a real six-dimensional symplectic vector space, and $\Lambda^{3} W^{*}$ the vector space of multilinear three-forms on $W$. Hitchin (2000) observes that for a three-form $\varpi \in \Lambda^{3} W^{*}$, we can identify a linear transformation $K_{\varpi}: W \mapsto W \otimes$ $\Lambda^{6} W^{*}$ by

$$
K_{\varpi}(w)=A(\iota(w) \varpi \wedge \varpi),
$$

where $A$ is the isomorphism $A: \Lambda^{5} W^{*} \cong W \otimes \Lambda^{6} W^{*}$ defined by the natural exterior product pairing $W^{*} \otimes \Lambda^{5} W^{*} \mapsto \Lambda^{6} W^{*}$, given $w \in W$ and the interior product $\iota(w) \varpi \wedge \varpi \in \Lambda^{5} W^{*}$. Hitchin defines $\lambda(\varpi) \in\left(\Lambda^{6} W^{*}\right)^{2}$ by

$$
\lambda(\varpi)=\frac{1}{6} \operatorname{Tr} K_{\varpi}^{2},
$$


and proceeds to use this Pfaffian ${ }^{3}$ to characterize non-degenerate elements of $\Lambda^{3} W^{*}$. In particular, depending on the sign of $\lambda(\varpi)$, Hitchin shows how $\varpi$ can be expressed as the sum of two decomposable three-forms. If $\lambda(\varpi)>0$, then $\varpi=\alpha+\beta$, where $\alpha, \beta$ are real decomposable three-forms and $\alpha \wedge \beta \neq 0$. If $\lambda(\varpi)<0$, then $\varpi=\alpha+\bar{\alpha}$, where $\alpha \in \Lambda^{3}\left(W^{*} \otimes \mathbb{C}\right)$ is a complex decomposable three-form and $\alpha \wedge \bar{\alpha} \neq 0$.

The relationship between $\Delta p, \zeta$ and $S$, given by (1.2), is the starting point for our geometric interpretation of vortex structures in Navier-Stokes turbulence. We observe that (1.2) can be expressed in terms of the vanishing of the threeform $\varpi \in \Lambda^{3} W^{*}$,

$$
\varpi=\Delta p \mathrm{~d} x_{1} \wedge \mathrm{d} x_{2} \wedge \mathrm{d} x_{3}-2\left(\mathrm{~d} u_{1} \wedge \mathrm{d} u_{2} \wedge \mathrm{d} x_{3}+\mathrm{d} u_{1} \wedge \mathrm{d} x_{2} \wedge \mathrm{d} u_{3}+\mathrm{d} x_{1} \wedge \mathrm{d} u_{2} \wedge \mathrm{d} u_{3}\right),
$$

on the graph of d $\boldsymbol{u}$, i.e. $\left.\varpi\right|_{\mathrm{d} u}=0$ (e.g. McIntyre \& Roulstone 2002, §5). It is the incompressibility condition that allows us to write (1.2) in terms of this three-form. Introducing the canonical symplectic form $\Omega=\mathrm{d} \boldsymbol{x} \wedge \mathrm{d} \boldsymbol{u} \in \Lambda^{2} W^{*}$, then a three-form $\varpi$ is said to be effective if $\Omega \wedge \varpi=0$ (Kushner et al. 2007) and we note that this condition is satisfied by (2.3).

Three invariants can be associated with the effective form (2.3): the tensor $K_{\varpi}$ (defined by $(2.1)$ ), the Pfaffian $\lambda(\varpi)$ (defined by (2.2)) and a metric $q_{\varpi}$ defined by

$$
q_{\varpi}(w, w) \equiv-\frac{1}{4} \perp^{2}(\iota(w) \varpi \wedge \iota(w) \varpi),
$$

where

$$
\perp \varpi=\iota\left(\frac{\partial}{\partial \boldsymbol{x}} \wedge \frac{\partial}{\partial \boldsymbol{u}}\right) \varpi,
$$

with $w \in W$. This metric is related to the tensor $K_{\varpi}$ via the symplectic structure: $q_{\varpi}(w, w)=\Omega\left(K_{\varpi} w, w\right)$ (see Banos 2002, §3.9).

The components of the metric derived from $(2.3)$ can be written as a $6 \times 6$ matrix, which can be expressed in block thus,

$$
q_{\varpi}=-2\left(\begin{array}{cc}
\frac{1}{2} \Delta p I & 0 \\
0 & I
\end{array}\right) .
$$

The components of the tensor $K_{\varpi}$ are found from $K_{\varpi}=\Omega q_{\varpi}$,

$$
K_{\varpi}=-2\left(\begin{array}{cc}
0 & I \\
-\frac{1}{2} \Delta p I & 0
\end{array}\right) .
$$

We find that $\operatorname{Tr} K_{\varpi}^{2}=-12 \Delta p$, and therefore the Pfaffian

$$
\lambda(\varpi)=\frac{1}{6} \operatorname{Tr} K_{\varpi}^{2}=-2 \Delta p .
$$

\footnotetext{
${ }^{3} \mathrm{~A}$ characteristic polynomial of the components of $\varpi$.
} 
Hence, when $\Delta p>0$ - which from (1.2) implies vorticity dominates over strainwe find $\lambda(\varpi)<0$. When $\lambda(\varpi)<0$, Hitchin shows how the real three-form $\varpi$ determines the structure of a complex vector space with a complex three-form on the real vector space $W$. In particular, when $\lambda(\varpi)<0$, the tensor

$$
J_{\varpi}=\frac{1}{\sqrt{-\lambda(\varpi)}} K_{\varpi}
$$

is an almost-complex structure and the real three-form $\varpi$ is the real part of the complex form

$$
\varpi^{\mathrm{c}}=\varpi+\mathrm{i} \hat{\varpi}
$$

with $\hat{\oplus}=\mathrm{i}(\bar{\alpha}-\alpha)$. From $(2.3)$ we find

$$
\begin{aligned}
\varpi & =\left(\mu_{1}+\mathrm{i} \nu_{1}\right) \wedge\left(\mu_{2}+\mathrm{i} \nu_{2}\right) \wedge\left(\mu_{3}+\mathrm{i} \nu_{3}\right)+\left(\mu_{1}-\mathrm{i} \nu_{1}\right) \wedge\left(\mu_{2}-\mathrm{i} \nu_{2}\right) \wedge\left(\mu_{3}-\mathrm{i} \nu_{3}\right) \\
& \equiv \alpha+\bar{\alpha},
\end{aligned}
$$

where $\mu_{i}=(\Delta p / 2)^{1 / 3} \mathrm{~d} x_{i}$ and $\nu_{i}=(\Delta p / 2)^{-(1 / 6)} \mathrm{d} u_{i}$, when $\Delta p>0$, in accordance with Hitchin's results. When $\Delta p<0$, and hence $\lambda(\varpi)>0$, we have a real decomposable structure

$$
\begin{aligned}
\varpi & =-\left(\mu_{1}+\nu_{1}\right) \wedge\left(\mu_{2}+\nu_{2}\right) \wedge\left(\mu_{3}+\nu_{3}\right)-\left(\mu_{1}-\nu_{1}\right) \wedge\left(\mu_{2}-\nu_{2}\right) \wedge\left(\mu_{3}-\nu_{3}\right) \\
& \equiv \alpha+\beta
\end{aligned}
$$

where $\mu_{i}=(-\Delta p / 2)^{1 / 3} \mathrm{~d} x_{i}$ and $\nu_{i}=(-\Delta p / 2)^{-(1 / 6)} \mathrm{d} u_{i}$. Banos (2002) shows how the pair $\left(q_{\varpi}, J_{\varpi}\right)$ is the starting point for constructing a generalized CalabiYau geometry.

\section{Discussion}

Equation (2.8) is our key result. It provides us with a generalization of the notion of elliptic and hyperbolic flows, as described by Weiss (1991), from the twodimensional case to the three-dimensional Navier-Stokes equations. In three dimensions, 'elliptic flows' are characterized by an almost-complex structure. In contrast to the results of McIntyre \& Roulstone (2002), in which vorticity - and hence the ellipticity of a Monge-Ampère equation - were salient features in large-scale atmospheric and oceanic flows (in which the rotation of the Earth is an important factor), we have shown how real and complex structures coexist in turbulent flows. Following Weiss (1991), we also note that $-\Delta p=\left(S_{i j} S_{j i}+\xi_{i j} \xi_{j i}\right)$, where $S_{i j}=(1 / 2)\left(u_{i, j}+u_{j, i}\right)$ and $\xi_{i j}=(1 / 2)\left(u_{i, j}-u_{j, i}\right)$ are the symmetric and antisymmetric parts of the velocity gradient tensor, $\nabla \boldsymbol{u}$, respectively. The invariants of the velocity gradient tensor have been much studied in connection with the analysis of vortical structures (Chong et al. 1990; Kida \& Miura 1998; Dubief \& Delcayre 2000), and we note that $\lambda(\varpi)$ is proportional to the second invariant of $\nabla \boldsymbol{u}$.

It may be possible to show that certain coherent or canonical flow states correspond to generalized Calabi-Yau structures: such structures exist when the almost-complex geometry satisfies certain integrability conditions (although these conditions are likely to correspond to severe restrictions on $\Delta p$; cf. Roulstone et al. in press). We remark that, while geometers are usually interested 
in classifying global structures, our results are local. That is, in general, different regions of the flow will be associated with real or complex structures in the sense defined in $\S 2$.

The authors wish to thank two anonymous referees for their helpful comments.

\section{References}

Banos, B. 2002 Nondegenerate Monge-Ampère structures in dimension 6. Lett. Math. Phys. 62, 1-15. (doi:10.1023/A:1021655609692)

Burgers, J. M. 1948 A mathematical model illustrating the theory of turbulence. Adv. Appl. Math. 1, 171-199. (doi:10.1016/S0065-2156(08)70100-5)

Chong, M. S., Perry, A. E. \& Cantwell, B. J. 1990 A general classification of three-dimensional flow fields. Phys. Fluids A 2, 765-777. (doi:10.1063/1.857730)

Douady, S., Couder, Y. \& Brachet, M. E. 1991 Direct observation of the intermittency of intense vorticity filaments in turbulence. Phys. Rev. Lett. 67, 983-986. (doi:10.1103/ PhysRevLett.67.983)

Dubief, Y. \& Delcayre, F. 2000 On coherent-vortex identification in turbulence. J. Turbulence 1, 1-22. (doi:10.1088/1468-5248/1/1/011)

Gibbon, J. D. 2002 A quaternionic structure in the three-dimensional Euler and ideal magnetohydrodynamics equations. Physica D 166, 17-28. (doi:10.1016/S0167-2789(02)00434-7)

Gibbon, J. D. 2007 Orthonormal quaternion frames, Lagrangian evolution equations, and the three-dimensional Euler equations. Russ. Math. Surveys 62, 535-560. (doi:10.1070/RM2007 v062n03ABEH004411)

Gibbon, J. D. 2008a The three-dimensional Euler equations: where do we stand? Physica D 237, 1894-1904. (doi:10.1016/j.physd.2007.10.014)

Gibbon, J. D. $2008 b$ Estimating intermittency in three-dimensional Navier-Stokes turbulence. Preprint. (http://arxiv.org/abs/0809.1811)

Gibbon, J. D. \& Holm, D. D. 2007 Lagrangian particle paths \& ortho-normal quaternion frames. Nonlinearity 20, 1745-1759. (doi:10.1088/0951-7715/20/7/010)

Gibbon, J. D., Fokas, A. \& Doering, C. R. 1999 Dynamically stretched vortices as solutions of the Navier-Stokes equations. Physica D 132, 497-510. (doi:10.1016/S0167-2789(99)00067-6)

Gibbon, J. D., Holm, D. D., Kerr, R. M. \& Roulstone, I. 2006 Quaternions and particle dynamics in the Euler fluid equations. Nonlinearity 19, 1969-1983. (doi:10.1088/0951-7715/19/8/011)

Hitchin, N. J. 2000 The geometry of three-forms in six dimensions. J. Differ. Geom. 55, 547-576.

Hoskins, B. J. \& Bretherton, F. P. 1972 Atmospheric frontogenesis models: mathematical formulation and solutions. J. Atmos. Sci. 29, 11-37. (doi:10.1175/1520-0469(1972)029< 0011:AFMMFA > 2.0.CO;2)

Kerr, R. M. 1985 Higher order derivative correlations and the alignment of small-scale structures in isotropic numerical turbulence. J. Fluid Mech. 153, 31-58. (doi:10.1017/S0022112085001136)

Kida, S. \& Miura, H. 1998 Identification and analysis of vortical structures. Eur. J. Mech. B/Fluids 17, 471-488. (doi:10.1016/S0997-7546(98)80005-8)

Kushner, A., Lychagin, V. \& Rubtsov, V. 2007 Contact geometry and non-linear differential equations, p. 496. Cambridge, UK: Cambridge University Press.

Larchevêque, M. 1990 Equation de Monge-Ampère et écoulements incompressibles bi-dimensionnels. C. R. Acad. Sci. Paris Ser. II 311, 33-36.

Larchevêque, M. 1993 Pressure field, vorticity field, and coherent structures in two-dimensional incompressible turbulent flows. Theor. Comp. Fluid Dyn. 5, 215-222. (doi:10.1007/ BF00271659)

Lychagin, V. V., Roubtsov, V. N. \& Chekalov, I. V. 1993 A classification of Monge-Ampère equations. Ann. Sci. Ecole. Norm. Sup. 26, 281-308.

McDuff, D. \& Salamon, D. 1998 An introduction to symplectic topology. 2nd edn, p. 486. Oxford, UK: Oxford University Press. 
McIntyre, M. E. \& Roulstone, I. 2002 Are there higher-accuracy analogues of semi-geostrophic theory? In Large-scale atmosphere-ocean dynamics, vol. II (eds J. Norbury \& I. Roulstone) Geometric methods and models, pp. 301-364. Cambridge, UK: Cambridge University Press.

Moffatt, H. K., Kida, S. \& Ohkitani, K. 1994 Stretched vortices - the sinews of turbulence; largeReynolds-number asymptotics. J. Fluid Mech. 259, 241-264. (doi:10.1017/S002211209400011X)

Roubtsov, V. N. \& Roulstone, I. 1997 Examples of quaternionic and Kähler structures in Hamiltonian models of nearly geostrophic flow. J. Phys. A 30, L63-L68. (doi:10.1088/0305$4470 / 30 / 4 / 004)$

Roubtsov, V. N. \& Roulstone, I. 2001 Holomorphic structures in hydrodynamical models of nearly geostrophic flow. Proc. R. Soc. Lond. A 457, 1519-1531. (doi:10.1098/rspa.2001.0779)

Roulstone, I. \& Sewell, M. J. 1997 The mathematical structure of theories of semigeostrophic type. Phil. Trans. R. Soc. Lond. A 355, 2489-2517. (doi:10.1098/rsta.1997.0144)

Roulstone, I., Banos, B., Gibbon, J. D. \& Roubtsov, V. N. In press. Kähler geometry and Burgers' vortices. Proc. Math. Inst. Natl Acad. Sci. Ukraine.

Siggia, E. D. 1981 Numerical study of small scale intermittency in three-dimensional turbulence. J. Fluid Mech. 107, 375-406. (doi:10.1017/S002211208100181X)

Vincent, A. \& Meneguzzi, M. 1994 The dynamics of vorticity tubes of homogeneous turbulence. J. Fluid Mech. 225, 245-254. (doi:10.1017/S0022112094003319)

Weiss, J. 1991 The dynamics of enstrophy transfer in two-dimensional hydrodynamics. Physica D 48, 273-294. (doi:10.1016/0167-2789(91)90088-Q) 